\title{
EDITORIAL
}

\section{LA PARAPLEGIE MASCULINE EN 1994}

Andrologie m'a fait l'honneur de me confier l'élaboration dans ce numéro d'une rubrique consacrée à la paraplégie, du fait sans doute d'une certaine expérience puisqu'il y a maintenant 25 ans que je m'occupe de paraplégie.

A cette époque, notre souci était surtout d'ordre vital : le paraplégique mourrait encore d'insuffisance rénale, d'escarres ou d'amylose.

Maintenant, le risque vital a pratiquement disparu en dehors de la période traumatique initiale pour le tétraplégique. Le confort et l'adaptation psychosociale sont devenus des objectifs prioritaires.

Il y a seulement 10 ans la principale qualité d'une vessie neurologique était de se bien vider. L'incontinence était non seulement négligée mais quasi-obligatoire. Aujourd'hui, tout paraplégique et même tout tétraplégique exigent une continence urinaire parfaite.

Le problème gênital a suivi cette même évolution, de secondaire il est devenu prioritaire. Tout paraplégique, quels que soient son niveau et son étiologie, veut avoir une érection, une éjaculation et des enfants.

Cette population neurologique n'est plus marginale en France puisqu'on y compte 35000 paraplégiques, dont 1800 nouveaux par an avec surtout 55\% de moins de 25 ans.

Seulement 5 à $10 \%$ de ces patients sont traités dans des centres ultra-spécialisés, autrement dit le paraplégique se considère de plus en plus comme un individu normal, susceptible d'être traité dans une structure médicale normale classique.

Il nous apparaît donc nécessaire de pouvoir transmettre à des non-paraplégistes une information suffisante sur nos connaissances et sur le traitement actuel des troubles génitosexuels de l'homme paraplégique.

Cette présentation est déjà suffisamment longue pour que dans ce numéro ne puissent être envisagées les perspectives d'avenir et la recherche.

Les différents auteurs représentent une expérience nationale puisqu'ils sont d'origine variée mais il existe entre eux une certaine unité de pensée et d'action car les paraplégistes représentent encore un milieu restreint où la communication reste facile.

Nous avons l'habitude d'échanger nos recettes et nous espérons pouvoir vous en faire profiter.

\author{
A. LERICHE \\ Professeur d'Urologie
}

